\title{
Interleukin 27 Signaling in Rheumatoid Arthritis Patients: Good or Evil?
}

\author{
Liang $\mathrm{Han}^{1 \dagger}$, Zhe Chen ${ }^{1+}$, Kun Yu ${ }^{2}$, Jiahui Yan ${ }^{1}$, Tingting $\mathrm{Li}^{1}$, Xin Ba ${ }^{1}$, Weiji Lin ${ }^{1}$, \\ Yao Huang ${ }^{1}$, Pan Shen ${ }^{1}$, Ying Huang ${ }^{1}$, Kai Qin ${ }^{1}$, Yinhong Geng ${ }^{3}$, Yafei Liu ${ }^{3}$, \\ Yu Wang ${ }^{1}$ and Shenghao $\mathrm{Tu}^{1 *}$ \\ ${ }^{1}$ Department of Integrated Traditional Chinese and Western Medicine, Tongji Hospital of Tongji Medical College of Huazhong \\ University of Science and Technology, Wuhan, China, ${ }^{2}$ Department of Cardiology, Tongji Hospital of Tongji Medical College \\ of Huazhong University of Science and Technology, Wuhan, China, ${ }^{3}$ Department of Nephrology, The First Affiliated Hospital \\ of Zhengzhou University, Zhengzhou, China
}

OPEN ACCESS

Edited by:

Cory Robinson,

West Virginia University, United States

Reviewed by:

Ganesan Ramamoorthi,

Moffitt Cancer Center, United States

Satoshi Kubo,

National Institutes of Health $(\mathrm{NIH})$,

United States

*Correspondence:

Shenghao Tu

shtu@tjh.tjmu.edu.cn

${ }^{\text {t}}$ These authors have contributed equally to this work and share first authorship

Specialty section: This article was submitted to Cytokines and Soluble Mediators in Immunity, a section of the journal

Frontiers in Immunology

Received: 30 September 2021 Accepted: 06 December 2021 Published: 04 January 2022

Citation:

Han L, Chen Z, Yu K, Yan J, Li T, Ba X, Lin $W$, Huang $Y$, Shen $P$, Huang $Y$, Qin $K$, Geng $Y$, Liu $Y$, Wang $Y$ and Tu S (2022) Interleukin 27 Signaling in Rheumatoid Arthritis Patients: Good or Evil?

Front. Immunol. 12:787252. doi: 10.3389/fimmu.2021.787252
The occurrence and development of rheumatoid arthritis (RA) is regulated by numerous cytokines. Interleukin 27 (IL-27) is a soluble cytokine that exerts biological effects by regulating the Janus tyrosine kinase $(\mathrm{JAK}) /$ signal transducer and activator of the transcription (STAT) signaling pathway via the $\mathrm{IL}-27$ receptor. $\mathrm{IL}-27$ is known for its pleiotropic roles in modulating inflammatory responses. Previous studies found that IL-27 levels are elevated in RA blood, synovial fluid, and rheumatoid nodules. Cellular and animal experiments indicated that IL-27 exerts multiple regulatory functions in RA patients via different mechanisms. IL-27 inhibits ectopic-like structure (ELS) formation and CD4 ${ }^{+} \mathrm{T}$ helper type 2 (Th2) cell, CD4 ${ }^{+}$T helper type 17 (Th17) cell, and osteoclast differentiation in RA, contributing to alleviating RA. However, IL-27 promotes Th1 cell differentiation, which may exacerbate RA synovitis. Moreover, IL-27 also acts on RA synovial fibroblasts (RAFLSs) and regulatory $T$ cells (Tregs), but some of its functions are unclear. There is currently insufficient evidence to determine whether IL-27 promotes or relieves RA. Targeting IL-27 signaling in RA treatment should be deliberate based on current knowledge.

Keywords: interleukin 27 (IL-27), rheumatoid arthritis, CD4+ $\mathrm{T}$ cells, synovial fibroblasts (FLS), osteoclasts (OCs), ectopic-like structure (ELS), rheumatoid nodules

\section{INTRODUCTION}

Rheumatoid arthritis (RA) is a systemic immune disease characterized by chronic symmetrical synovitis inflammatory polyarthritis of the small joints (1). In addition to arthritis, RA causes various extra-articular complications, such as RA-associated interstitial lung disease (RA-ILD) and skin damage $(2,3)$. Specific environmental and genetic factors cause immune system disorders in RA patients and determine the occurrence and development of RA (4).

Various immune cells and multiple cytokines produced by immune cells exert essential roles in the development and disease progression of RA. Interleukins (ILs) are a class of cytokines widely involved in immune system modulation and are implicated in RA. Two $\mathrm{CD} 4^{+} \mathrm{T}$ cell subsets, $\mathrm{T}$ helper 1 cells (Th1) and T helper 17 cells (Th17), have long been considered risk factors for RA development (5). In contrast, T helper 2 cells (Th2) and regulatory T cells (Tregs) are decreased in 
number and exhibit functional impairment in RA. IL-1 and IL17, which are secreted by Th1 and Th17 cells, respectively, have been reported to be proinflammatory factors in RA synovial membranes. In addition, IL-15, secreted by macrophages, promotes $\mathrm{T}$ cells in RA synovial membrane tumor necrosis factor (TNF)- $\alpha$ production in a cell contact-dependent manner (6). The cytokine network, constituted by multiple cytokines and feedback loops, subtlety regulates the immune response and inflammation in complex ways and is involved in RA development (7).

IL-27 is a pleiotropic immunoregulatory cytokine that was first identified in 2002 (8). Since the discovery of IL-27, its immune modulatory function has been gradually elucidated by numerous studies. An association between IL-27 single-nucleotide polymorphisms (SNPs) and genetic susceptibility to RA has been described (9). Abnormal IL-27 levels have been well demonstrated in previous studies, and IL-27 participates in RA development via multiple pathways (10-12). Earlier studies found that IL-27 primarily affects the differentiation of helper T cell subsets (13). However, more recent studies suggest that IL-27 might contribute to the pathogenesis of RA through additional direct and indirect regulatory pathways. Current studies have shown that IL-27 is extensively and profoundly involved in immunological imbalance in RA. Furthermore, IL-27 is a potential therapeutic target for the treatment of immune-related diseases. This review discusses updates on the regulatory effects of IL-27 on immune cells and RA development. We expect that our review may provide ideas for future studies, which would promote increased awareness of the relationship between RA and IL-27.

\section{THE COMPONENTS AND EXPRESSION OF IL-27 AND THE IL-27 RECEPTOR (IL-27R)}

The IL-27 receptor (IL-27) is a member of the IL-12 and IL-6 cytokine families and was identified two decades ago $(8,14,15)$. IL-27 is a heterodimer with p28, and Epstein-Barr virus induced 3 (EBI3) subunits that are encoded by IL27 and EBI3, respectively (Figure 1). The IL-27 p28 subunit is homologous to IL-12 p35 and IL-6 (16). EBI3 was first identified in B lymphocytes transfected with Epstein-Barr virus (EBV), and it is homologous to IL-12 p40 (17). However, the two subunits can exist independently. The IL-27 p28 subunit, also known as IL-30, can be secreted alone (18). In this review, we use the designation "IL-27 p28 subunit" instead of "IL-30" because the name of the gene encoding the IL-27 p28 subunit protein is IL-27 according to HGNC guidelines (8).

Existing studies suggest that recombinant IL-27 p28 blocks the activity of cytokines, including IL-6, IL-11, and IL-27 (16), which suppresses hepatic injury and alleviates experimental autoimmune uveitis (19). However, other studies found that the conformation of IL-27 p28 used in some studies was incorrect. IL-27 p28 can bind with soluble IL-6R $\alpha$ and signal in the presence of a more appropriate conformation. IL-27 p28 can even signal independently at high concentrations $(20,21)$. This feature of IL-27 p28 raises a concern about IL-27, as we do not know whether IL-27 or IL-27 p28 is performing these biological functions. Further investigation is warranted to determine whether another subunit of IL-27, EBI3, can exert some of its biological functions independently. Determining the answers to these questions could help to better understand the roles of IL-27 in diseases.

IL-27R, a heterodimer, is a member of the class I cytokine receptor family, which is composed of $\alpha$ subunits of the cytokine binding protein and $\beta$ subunits of the signal transduction protein (Figure 1) $(14,15,22)$. The two subunits, IL-27R $\alpha$, also known as T cell cytokine receptor (TCCR) or WSX-1, and glycoprotein 130 (gp130) are composed of IL-27R, and they are both members of the immunoglobulin superfamily. Gp130 is a receptor subunit that is shared by IL- 6 family cytokines. Both IL-27R $\alpha$ and gp130 are necessary for IL-27 signal transduction. Memory CD8 ${ }^{+} \mathrm{T}$ cells are not responsive to IL-27 stimulation because they downregulate the expression of gp130 (23). Moreover, research has indicated that IL-27R $\alpha$ is capable of forming homodimers and activates Janus tyrosine kinase (JAK)/signal transduction and activator of transcription (STAT) signaling $(24,25)$. Additionally, soluble IL-27R $\alpha$ is able to inhibit IL-27 signaling (26). Similar to IL-27, these features of IL-27R also influence our understanding of IL-27 signaling in diseases.

IL-27R $\alpha$ is expressed by several varieties of immune cells and non-immune cells, including $\mathrm{T}$ and $\mathrm{B}$ lymphocytes, NK cells, monocytes, macrophages, and hepatocytes (27). It has been reported that lymphoid tissues, including the spleen, thymus, and lymph nodes, also express high levels of IL-27R $\alpha$ (28). Most cells express gp130, and its expression levels are related to cellular status (29). Cells in different states may express different levels of IL-27. Cell activation causes $\mathrm{CD} 4^{+}$T cells to express increased IL-27R $\alpha$ but causes NK cells to express reduced levels, which influences cellular responses to IL-27 signaling.

\section{SOURCES AND SIGNALING PATHWAYS OF IL-27}

IL-27 is a soluble-secreted cytokine, so understanding its cellular sources is important. The two subunits of IL-27 are expressed by many immune cells, such as monocytes/macrophages, lymphocytes, activated dendritic cells (DCs), plasma cells, natural killer $(\mathrm{NK})$ cells, and other non-immune cells, such as placental trophoblasts and endothelial cells $(30,31)$. Specific signaling stimulation also regulates IL-27 expression. Dibra et al. found that coexisting Toll-like receptor (TLR) 9 signaling from macrophages and CD3 signaling from $\mathrm{T}$ cells stimulate the expression of IL-27 p28 in splenic cells (32). Liu et al. determined that lipopolysaccharide (LPS) and interferon- $\gamma$ stimulation promotes IL-27 p28 expression in macrophages (30). Moreover, in DCs, expression of EBI3 was induced by TLR signaling via nuclear factor kappa B (NF- $\mathrm{KB}$ ) (33).

As a soluble cytokine, IL-27 primarily acts as a first messenger and mediates IL-27-induced signal transduction by binding to cell membrane-bound IL-27R. IL-27R typically activates the JAK family, and the JAK family-induced signaling pathway is considered the classical apex of the IL-27 signaling cascade (34). The cytoplasmic region of IL-27R $\alpha$ has a Boxl motif and 


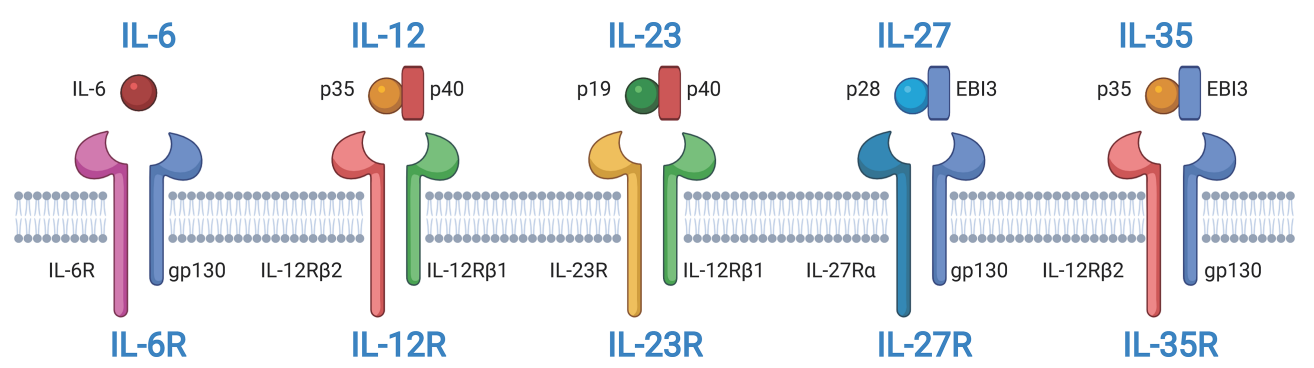

FIGURE 1 | Structures of interleukin-12 (IL-12) and IL-6 cytokine families and their receptors. IL-6, IL-12, IL-23, IL-27, and IL-35 belong to IL-12 and IL-6 cytokine families. IL-12, IL-27, and IL-35 are composed of two subunits. IL-12 and IL23 share the common subunit p40. IL-12 and IL-35 share the common subunit p35. IL27 and IL-35 share the common subunit Epstein-Barr virus induced 3 (EBI3). All receptors of IL-6, IL-12, IL-23, IL-27, and IL-35 are heterodimers. Some subunits are shared among receptors. IL-6R, IL-27R, and IL-35R share the common receptor subunit gp130. IL-12 and IL-23 share the common receptor subunit IL-12R 31 . $\mathrm{IL}-12 \mathrm{R}$ and IL-35R share the common receptor subunit IL-12R $\beta 2$. The figure was created with BioRender.com.

an SH domain, which is related to JAK1 and JAK2 and may assist in signal transduction (35). Gp130 is also associated with the JAK family members JAK1, JAK2, and tyrosine kinase (TYK) 2. The IL-27 signaling pathway can be activated by IL-27 and its receptor IL-27R in different cell types and in different cellular states (Figure 2). In mast cells, IL-27 treatment leads to STAT3 phosphorylation and increases mRNA levels of IL- $1 \alpha$, IL-1 $\beta$, IL18 , and TNF- $\alpha$ (28). In primary monocytes, IL-27 stimulation induces the expression of IL- $6, \mathrm{C}-\mathrm{X}-\mathrm{C}$ motif chemokine (CXCL) 10, C-C motif chemokine ligand (CCL) 3 , CCL4, and TNF- $\alpha$, accompanied by phosphorylation of STAT1 and STAT3 (28, 36). IL-27 also increases the phosphorylation of STAT1 and STAT3 in macrophages (37). In germinal center B cells, IL-27 induces weak phosphorylation of STAT1 and STAT3. However, IL-27 significantly promotes the phosphorylation of STAT1 and STAT3 and increases the expression of the transcription factor T-bet in naive and memory B cells. In anti-Ig-stimulated-naive or memory B cells, IL-27 also induces CD54, CD86, and CD95 expression. Moreover, IL-27 increases the proliferation of antiIg-activated naive $\mathrm{B}$ cells and anti-CD40-activated naive and germinal center B cells but not of CD40-activated memory B cells. In $\mathrm{CD}^{+} \mathrm{T}$ cells, IL-27 induces expression of the transcription factor T-bet in a STAT1-dependent and STAT1independent manner, which is crucial for Th1 polarization. IL-27 activates different JAK family members and downstream signaling pathways in different cell types and cell states, which regulates the signaling and biological pattern of immune cells. However, the exact roles and mechanisms of IL-27-induced JAK signal transduction in different cells remain unclear, and further studies are needed.

\section{IL-27 IS RELATED TO THE PROGRESSION OF RA}

\section{Elevated IL-27 Levels Are Detected in RA Patients}

Many studies have demonstrated that there are higher levels of IL-27 in RA peripheral circulation. Wong et al. found that RA patients exhibited higher plasma concentrations of IL-27 than healthy controls (10). Similarly, two studies by Shen et al. and Lai et al. found that IL-27 in RA serum is significantly increased, and levels of serum IL-27 are positively correlated with disease activity score 28 (DAS-28), indicating that high disease activity of RA is accompanied by high IL-27 levels $(38,39)$. Moreover, Shen et al. suggested that RA-ILD patients also displayed high levels of serum IL-27 (38). After leflunomide treatment, RA

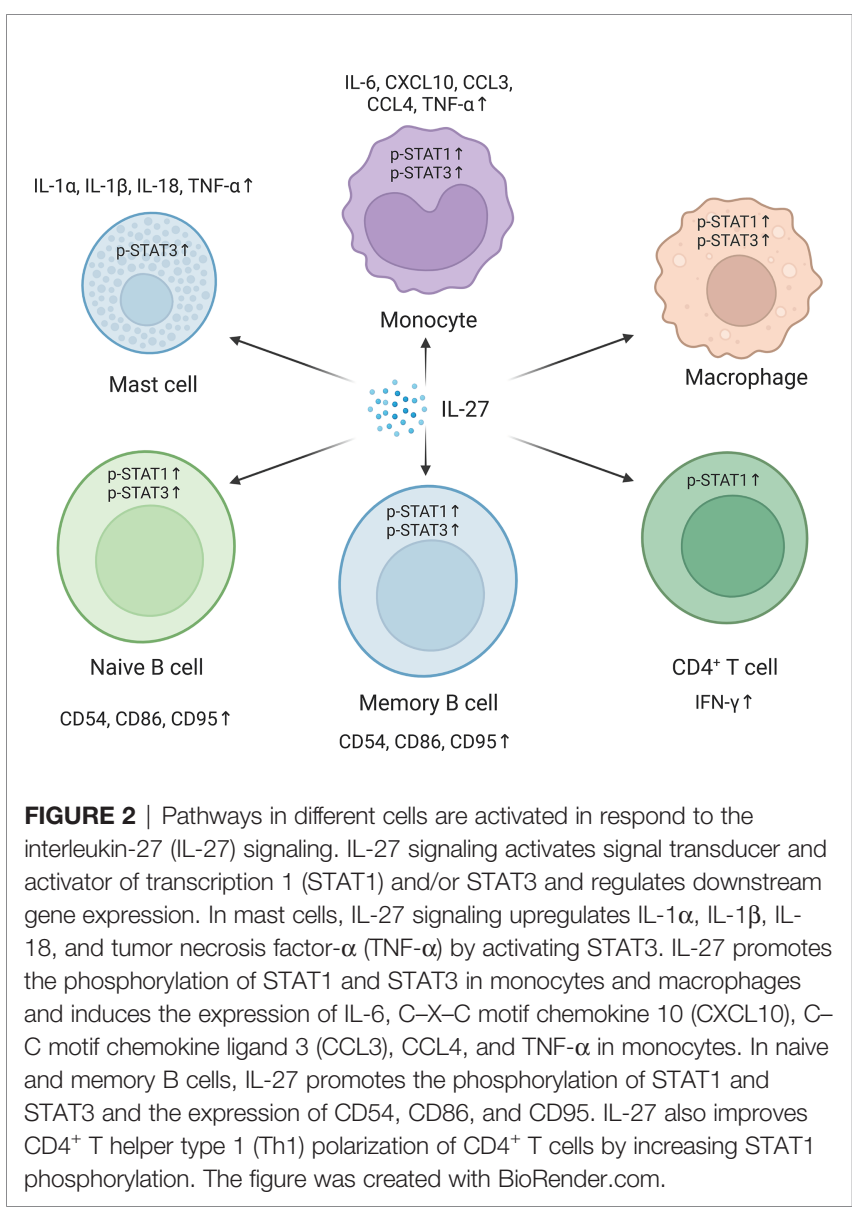


patients also exhibited decreased serum IL-27 levels (39). However, Tanida et al. suggested that plasma IL-27 levels in RA and osteoarthritis (OA) patients were comparable (11). In summary, the above studies indicated that the observed increased IL-27 levels reflect a certain degree of inflammatory status in RA patients.

Rheumatoid nodules are a common extra-articular manifestation of RA. Rheumatoid nodules are frequently present in subcutaneous pressure points and occasionally in visceral organs, such as the lung and heart (40-42). The pathological mechanism of rheumatoid nodules has been identified as granulomas driven by Th1 cells (3). Recently, Millier et al. determined that IL-27 levels in RA rheumatoid nodules are higher than that in RA synovial membranes and even higher than that in synovial membranes from end-stage RA (12). This means that rheumatoid arthritis might be a crucial source of IL-27 in RA. It is known that the occurrence of rheumatoid nodules is related to RA severity. Early RA patients who have subcutaneous rheumatoid nodules exhibit more rapid joint destruction and higher rates of hospital admission $(43,44)$. However, the level of IL-27 in rheumatoid nodules was studied only by one research group, and there is no greater independent evidence to document this. Therefore, it should be treated with caution. Further studies should keep exploring the level of IL-27 in rheumatoid nodules and whether IL-27 from rheumatoid nodules contributes to sustained systemic and synovial inflammation.

Studies comparing IL-27 levels in the joint cavity between RA and OA are lacking. However, increased IL-27 levels have been detected in both RA synovial membranes and synovial fluids compared to OA tissues $(11,12)$. Moreover, mRNA expression of the IL-27 receptor is also increased in RA synovial membranes. Increased IL-27 levels indicate that there are correlations between IL-27 and RA, meaning that the IL-27 signaling pathway might contribute to synovitis in RA. However, the possibility cannot be ruled out that IL-27 might be an innocent bystander in RA (12).

\section{Association Between IL-27 Gene Polymorphisms and RA}

It is well known that the occurrence of complex diseases is usually affected by genetic environmental factors. An SNP is a widespread and crucial form of genetic variation that has been used to explore disease susceptibility with respect to genetic factors (45). In RA, more than 100 SNPs are related to RA susceptibility factors according to genome-wide association study (GWAS) analysis (46). An association of the IL-27 SNP with genetic susceptibility to RA in Chinese Han and Polish populations has been described, which implies that IL-27 may be associated with the occurrence and development of RA $(9,47)$.

\section{IL-27 Treatment Exacerbates or Alleviates Arthritis in Animal Experiments}

Although it is unknown how exogenous IL-27 affects the progression of RA patients, validation from an arthritis animal model could help to clarify this. IL-27 plays paradoxical roles in arthritis animal models. Niedbala et al. first explored the role of IL-27 in arthritis mice and found that arthritis in IL-27-treated collagen-induced arthritis (CIA) mice was relieved (48). The intra-articular overexpression of IL-27 also attenuates arthritis severity in CIA mice (49). However, IL-27 treatment worsens arthritis in proteoglycan-induced arthritis (PGIA) mice (50). These seemingly contradictory results are reasonable when considering different pathological mechanisms in different arthritis models. Specifically, arthritis in CIA mice is primarily dominated by Th17 cell expansion but Th1 cells in PGIA mice $(51,52)$. Due to the distinct pathogenesis and pleiotropy of IL-27, the responses of different arthritis animal models are diametrically opposed. Evidences from arthritis animal models suggests that rather than a bystander, IL-27 may also be involved in the progression of RA.

\section{CROSS TALK BETWEEN IL-27 SIGNALING AND RA PATHOLOGIES}

Given the available studies, we can preliminarily determine that IL-27 signaling could influence RA development by regulating $\mathrm{CD}^{+} \mathrm{T}$ cell differentiation, inhibiting monocytes/macrophages and osteoclasts in the joint cavity, interrupting synovial ectopic lymphoid structure (ELS) interactions with Th17 cells, and regulating RA synovial fibroblast (RA-FLS)-mediated inflammation. However, some conclusions from different studies are conflicting, and clinical samples or arthritis models were not utilized in some studies. To clearly present and discuss the mechanisms of IL-27 signaling and RA pathologies, a table is provided (Table 1).

\section{IL-27 Regulates CD4 ${ }^{+}$T Cell Differentiation}

$\mathrm{CD}^{+} \mathrm{T}$ cells play a pivotal role in RA physiopathology (69). Th1 cell infiltration is traditionally believed to be crucial for RA pathogenesis due to inadequate knowledge of the $\mathrm{CD}^{+} \mathrm{T}$ cell subpopulation (69). With advances in $\mathrm{CD}^{+} \mathrm{T}$ cell research, Th17 cells have been identified as a separate $\mathrm{CD} 4^{+}$cell subpopulation that is closely related to autoimmune diseases $(70,71)$. RA has also been interpreted as a Th17-driven autoimmune disease (71). There was a positive correlation between the DAS- 28 score and Th17 cells but not the Th1 cell proportion in RA peripheral blood (71). Moreover, when discussing $\mathrm{CD}^{+} \mathrm{T}$ cells in RA, Tregs also deserve our attention. Tregs exert pivotal roles in the maintenance of immunosuppressive activity (72). However, impaired Treg function in RA patients has been observed and is considered a key pathological change (73).

The regulatory roles of IL- 27 on the $\mathrm{CD} 4^{+} \mathrm{T}$ cell subpopulation can be summarized as follows: IL-27 promotes Th1 cells but restrains Th2 and Th17 cells (Figure 3). IL-27 induces Th1 cells by upregulating T-box expressed in T cell (T-bet) expression via STAT1 activation and activating TYK2/mitogen-activated protein kinase (MAPK)/T-bet and leukocyte function-associated antigen (LFA)-1/intercellular adhesion molecule (ICAM)-1/extracellular signal-regulated kinase (ERK) $1 / 2$ signaling pathways $(53,54)$. Moreover, IL-27 inhibits the expression of GATA-binding protein 3 (GATA-3) in naive $\mathrm{CD}^{+}{ }^{+} \mathrm{T}$ cells via STAT1 activation, 
TABLE 1 | Effects and functions of IL-27 in RA different tissues and cells.

\begin{tabular}{|c|c|c|c|c|}
\hline Targets of IL-27 & $\begin{array}{l}\text { Sources of } \\
\text { evidence }\end{array}$ & Effects & Mechanisms & References \\
\hline $\mathrm{CD}^{+} \mathrm{T}$ cell & $\begin{array}{l}\text { Animals and } \\
\text { cells }\end{array}$ & Uncertain & $\begin{array}{l}\text { IL-27 promotes Th1 development and inhibits Th17 cell differentiation. However, the role of IL-27 } \\
\text { in RA Treg development remains unclear according to the current research. }\end{array}$ & $(48,53-63)$ \\
\hline Osteoclasts & $\begin{array}{l}\text { Clinical samples } \\
\text { and animals }\end{array}$ & Protective & IL-27 inhibits osteoclast differentiation by inhibiting RANK/RANKL signaling pathway. & $(65,66)$ \\
\hline ELS & $\begin{array}{l}\text { Clinical samples } \\
\text { and animals }\end{array}$ & Protective & IL-27 inhibits the formation of ELS by inhibiting Pdp ${ }^{+}$Th17 cells. & $(67,68)$ \\
\hline
\end{tabular}

Th1, CD4 $4^{+}$Thelper type 1; Th17, CD4 $4^{+}$Thelper type 17; MNC, mononuclear cell; RANK, receptor activator of nuclear factor kappa-B (RANK); RANKL, RANK legend; RA-FLS, rheumatoid arthritis synovial fibroblast; ELS, ectopic-like structure.

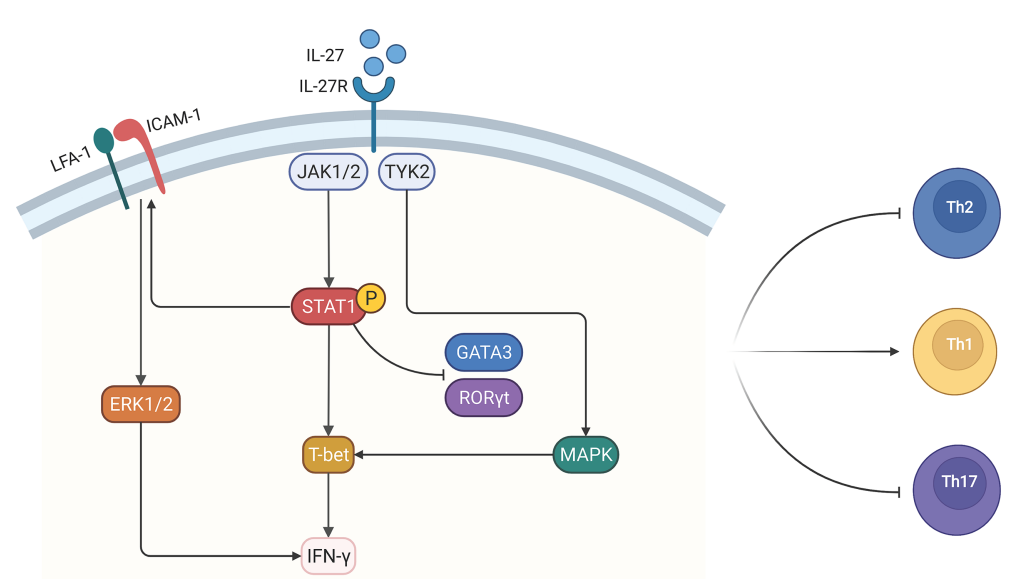

Naive $C D 4^{+} T$ cell

FIGURE 3 | Interleukin-27 (IL-27) promotes CD4+ T helper type 1 (Th1) cell differentiation and inhibits Th2 and Th17 cell development in CD4+ T cells. IL-27 promotes Th1 development via multiple signaling pathways. In CD4 ${ }^{+}$T cells, IL-27 binds to IL-27 receptor and activates Janus tyrosine kinase 1 (JAK1), JAK2, and tyrosine kinase 2 (TYK2). Subsequently, phosphorylated signal transducer and activator of transcription 1 (STAT1) activates T-bet, and this drives naive CD4 ${ }^{+}$T cell to skew toward Th1 cells. STAT1 phosphorylation also promotes Th1 development via leukocyte function-associated antigen 1 (LFA-1)/intercellular adhesion molecule 1 (ICAM-1) and extracellular signal-regulated kinase (ERK)1/2 signaling pathway. In addition, Th1 differentiation can be improved by the TYK2/MAPK/T-bet signaling pathway, which is STAT1 independent. STAT1 phosphorylation induced by IL-27 inhibits GATA-binding protein 3 (GATA-3) and orphan receptor gamma t (ROR rt) via a STAT1-dependent manner, which blocks naive $\mathrm{CD}_{4}^{+} \mathrm{T}$ cell differentiate into Th2 and Th17. The figure was created with BioRender.com.

which ultimately inhibits Th2 differentiation (55). For Th17 cells, IL-27 downregulates IL-17 expression via retinoic acid-related orphan receptor gamma $\mathrm{t}(\mathrm{ROR} \gamma \mathrm{t})$ inhibition by activating the STAT1 signaling pathway (56).

The role of IL-27 in Tregs exhibits double-sided and confusing effects. On the one hand, several studies have shown that the IL-27 signaling pathway promotes proinflammatory functions via Treg inhibition. IL-27 inhibits Treg cell generation and the expression of CD25 and cytotoxic T lymphocyte-associated antigen (CTLA)-4 under in vitro conditions (57). Another in vitro experiment demonstrated that IL-27 suppresses the development of induced Tregs in a STAT1-independent manner (58). In addition, blocking the IL-27 signaling pathway also alleviates mouse colitis by limiting naive $\mathrm{CD}^{+}{ }^{+} \mathrm{T}$ cell to Treg conversion (59). On the other hand, IL-27 exerts anti-inflammatory effects in Tregs in several disease conditions. Nguyen et al. reported that Tregs inhibit effector $\mathrm{T}$ cell proliferation in experimental allergic airway inflammation in a lymphocyte activation gene (LAG)3-dependent manner when the IL-27 signaling pathway is activated, although Treg proliferation is also suppressed (60). Do et al. demonstrated that Treg-specific IL27R $\alpha$ deletion did not alter Treg development under in vivo conditions (61). Further in-depth research from Do et al. found that IL-27 signaling activation is essential for the suppressive function and stability of Foxp $3^{+}$Tregs in an encephalomyelitis (EAE) mouse model, which was accompanied by LAG3 upregulation. Similar to the above studies, the IL-27/LAG3 axis was found to enhance Foxp $3^{+}$Treg-suppressive function during intestinal inflammation (62). However, a study from Batten et al. indicated that Tregs from Il27ra deletion mice also inhibited effector $\mathrm{T}$ cell proliferation, indicating that the IL-27 signaling pathway is 
not essential for Treg-suppressive functions under physiological conditions (63). In an arthritis model, IL-27 treatment affected the expression of IL-17 but not Foxp3 in CIA mouse spleens (48). Regrettably, at present, no experiment has determined whether and how IL-27 signaling affects Tregs in the synovitis microenvironment in RA patients. In summary, the IL-27 signaling pathway has dual effects on Tregs. It seems that IL-27 signaling is required for Treg-suppressive functions under disease conditions in vivo. However, direct evidence for this is needed in RA patients and model mice.

\section{IL-27 Inhibits Inflammation and Differentiation of Monocytes/Macrophages in RA Joints}

It seems likely that IL-27 protects RA patients from joint inflammation and joint destruction via the monocyte/ macrophage lineage according to in vitro experiments. Tanida et al. found that IL-27 in the joint cavity appears to be produced by $\mathrm{CD} 14^{+}$mononuclear cells (MNCs) as determined by analyzing RA synovial fluid because $\mathrm{IL}^{+} 7^{+}$cells are primarily localized in $\mathrm{CD}_{14}{ }^{+} \mathrm{MNCs}$ (11). In addition, there were more IL$27^{+}$MNCs in RA synovial membranes than in OA synovial membranes. Considering that CD14 is predominantly expressed in monocytes, macrophages, and DCs, we speculate that the monocyte/macrophage lineage in the joint cavity constitutes the main source of IL-27 in synovial fluids (64). A study from Tanida et al. also demonstrated that levels of IL-27 in the joint cavity are negatively correlated with IL-17 levels, indicating that IL-27 inhibits inflammation mediated by Th17 cells in the joint cavity (11).

Osteoclasts are supposed to differentiate from CD $14^{+}$ monocytes $(74,75)$. Osteoclastic bone erosion plays a dominant role in RA joint destruction. The receptor activator of nuclear factor kappa-B (RANK)/RANK ligand (RANKL) signaling pathway plays crucial roles in osteoclast differentiation. Activation of IL-27 signaling strongly depresses osteoclast differentiation by inhibiting RANK expression, RANKL-mediated MAPK, and NF- $\mathrm{KB}$ signaling pathways and by inhibiting RANKL-mediated nuclear factor of activated $\mathrm{T}$ cell (NFATC) 1 induction (65). This inhibitory effect is correlated with IL-27 receptor levels. Compared to human-derived monocytes, mouse-derived monocytes express lower levels of WSX-1, which might be the reason that $\mathrm{CD} 14^{+}$monocyte differentiation into osteoclasts was moderately inhibited. In addition, IL-27 downregulates RANKL expression in $\mathrm{CD}^{+} \mathrm{T}$ cells in part through STAT3, which contributes to the inhibition of $\mathrm{CD}^{+} 4^{+}$monocyte differentiation (66). In short, IL-27 negatively regulates osteoclast formation and bone resorption (75), which is beneficial for arresting the progression of bone destruction.

\section{Is IL-27 a Pro- or Anti-Inflammatory Agent in RA-FLS?}

It seems that the effect of IL-27 on RA-FLSs is paradoxical according to in vitro experiments. First, IL-27 increases the expression of adhesion molecules, including ICAM-1; vascular cell adhesion molecule (VCAM)-1; inflammatory chemokines; including CCL2, CXCL9, and CXCL10; and matrix metalloproteinase (MMP) 1 (10). Moreover, a combination of IL-27 and TNF- $\alpha$ or IL- $1 \beta$ treatment significantly upregulates adhesion molecules and chemokines. The above results indicate that IL-27 inhibits the RA-FLS-mediated inflammatory response. However, another in vitro study demonstrated that a combination of IL-27 and TNF- $\alpha$ or IL-17A treatment decreased RA-FLS expression of IL-6 and CCL20 (11). Taken together, the two studies indicate that IL-27 may exert anti- or proinflammatory roles on RA-FLSs in different environments. The regulation of RA-FLSs by IL-27 is complicated and might be similar to that of $\mathrm{CD}^{+} \mathrm{T}$ cells. The unique regulatory characteristics of IL-27 in RA-FLSs should be noted and explored in future research.

\section{IL-27 May Break the Vicious Cycle Between ELS and Th17 Cells}

It is known that ELS represents a structure of lymphocyte clusters in non-lymphoid tissues, primarily consisting of $\mathrm{B}$ cells, T cells, and DCs. ELS is closely related to both cellular immunity and humoral immunity (67). The synovial membrane pathological feature in approximately $40 \%$ of RA patients is follicular synovitis with ELS (76). $\mathrm{Pdp}^{+} \mathrm{Th} 17$ cells contribute to ELS formation (77-79). Of note, IL-27 regulates the formation and development of ELS (67). A previous study also found that in RA patients and adjuvant-induced arthritis (AIA) mice, IL-27 signaling inhibits $\mathrm{Pdp}^{+}$Th17 cells, which is linked to ELS formation (68). In light of this, IL-27 may break the proinflammatory positive feedback between ELS and $\mathrm{Pdp}^{+}$ Th17 cells. However, not all RA synovitis cases exhibit ELS. Comprehensive and stratified evaluation should be performed to determine the function of IL-27 in RA synovitis.

\section{IL-27 SIGNALING REPRESENTS AN RA THERAPEUTIC TARGET: FUTURE POTENTIAL AND CURRENT CHALLENGES}

The IL-27 signaling pathway has not been clinically investigated as a potential target for disease treatments except in tumors. Recently, SRF388, a targeted IL-27 p38 antibody, has been evaluated for its safety and therapeutic potential for the treatment of advanced solid tumors in a phase 1, first-inhuman, dose-escalation, and expansion study (NCT04374877). Solid tumor animal models and cellular experiments with bioinformatics research have demonstrated that blocking IL-27 signaling is beneficial for the treatment of hepatocellular carcinoma and renal cell carcinoma $(80,81)$. The mediumterm results of a phase I clinical trial demonstrated that SRF388 inhibited STAT3 phosphorylation in 12 recruited tumor patients with mild adverse reactions (82). These results indicate that inhibiting IL-27 signaling via SRF388 might represent an alternative tumor treatment.

However, IL-27, a pleiotropic cytokine, yielded confusing results when attempting to alleviate RA patients by intervening 
in IL-27 signaling. It is difficult to see that the roles of IL-27 in regulating RA pathology are many and even contradictory. Evidence from animal studies suggests that IL-27 exerts proor anti-inflammatory roles in different arthritis animal models. Conflicting results have also been observed in numerous cellular experiments. Considering the limitations of animal and cellular studies, it is not currently possible to conclude whether addition or blocking of IL-27 would treat RA according to only one or multiple laboratory studies. The roles of IL-27 in cells or tissues related to RA pathology should continue to be explored using cellular and animal studies in the future. However, further studies on IL-27's levels and roles in RA blood, joint cavities, and rheumatoid nodules by detecting clinical specimens from RA are also important. Alejandro et al. focused on the roles of IL27 in RA patients with follicular synovitis and found that agents regulating IL-27, such as SRF388, might only be suitable for RA patients with specific clinical and pathologic characteristics (68).

\section{CONCLUSIONS}

Overall, the intricate links between RA immune regulation and inflammatory responses are being actively explored but are still not fully understood. Many cytokines exert beneficial or detrimental effects on RA patients by regulating immune and inflammatory responses. Among these cytokines, IL-27 is undoubtedly one of the most controversial cytokines for RA due to its pleiotropism. The awareness of immunity and inflammation is expanded while exploring the biological functions of IL-27. The pleiotropic regulatory functions of IL27 serve as a reminder that inflammation involves a series of continuously changing states rather than simply a resting or constant state. IL-27 might be a cytokine that promotes an inflammatory state.

In the present article, we systematically reviewed and discussed the associations between RA and IL-27 by referencing previous studies. IL-27 levels are generally elevated in blood, synovial fluids, synovial fibroblasts, and even rheumatoid nodules. Nevertheless, it remains unclear whether higher levels of IL-27 are beneficial or harmful to RA patients. According to experimental research, IL-27 alleviates RA progression by inhibiting ELS formation and osteoclast

\section{REFERENCES}

1. Guo Q, Wang Y, Xu D, Nossent J, Pavlos NJ, Xu J. Rheumatoid Arthritis: Pathological Mechanisms and Modern Pharmacologic Therapies. Bone Res (2018) 6:15. doi: 10.1038/s41413-018-0016-9

2. Cassone G, Manfredi A, Vacchi C, Luppi F, Coppi F, Salvarani C, et al. Treatment of Rheumatoid Arthritis-Associated Interstitial Lung Disease: Lights and Shadows. J Clin Med (2020) 9(4):1082. doi: 10.3390/jcm9041082

3. Hessian PA, Highton J, Kean A, Sun CK, Chin M. Cytokine Profile of the Rheumatoid Nodule Suggests That It Is a Th1 Granuloma. Arthritis Rheum (2003) 48:334-8. doi: 10.1002/art.10776

4. Deane KD, Demoruelle MK, Kelmenson LB, Kuhn KA, Norris JM, Holers VM. Genetic and Environmental Risk Factors for Rheumatoid Arthritis. Best Pract Res Clin Rheumatol (2017) 31:3-18. doi: 10.1016/j.berh.2017.08.003 differentiation. However, other laboratory evidence indicates that the roles of $\mathrm{IL}-27$ in $\mathrm{CD}^{+}{ }^{+} \mathrm{T}$ cells and RA-FLSs are complex and even conflicting, so it is difficult to draw a definitive conclusion regarding IL-27's functions.

Considering that widely used arthritis animal models do not fully reflect the pathological characteristics of RA, use of clinical samples from RA should be considered in future studies. IL-27 might exert different roles in different RA patients; thus, the clinical characteristics, including demographic information, laboratory tests, and medication history, of RA patients should be recorded in detail. Overall, the existing evidence suggests that IL-27 exerts both pro- and anti-inflammatory effects in different ways. It is hard to say whether activating or inhibiting the IL-27 signaling pathway would be effective as an RA treatment. It is possible that some RA patients would benefit from IL-27 signaling pathway activation, while others may benefit from pathway inhibition, and still others might be unsuitable for treatment targeting the IL-27 signaling pathway. Therefore, careful attention should be given to IL-27 signaling-targeted therapy for RA to clearly understand how IL-27 signaling works in RA patients and whether RA patients would benefit from intervening in IL-27 signaling.

\section{AUTHOR CONTRIBUTIONS}

ST contributed to the conception of this review. LH wrote the manuscript. LH, ZC, and KY revised the manuscript. LH and KY designed and illustrated the figures. ZC, JY, TL, WL, XB, and PS performed the literature search and interpretation. ZC, YG, YL, $\mathrm{YaH}, \mathrm{YiH}, \mathrm{KQ}, \mathrm{YW}$, and $\mathrm{ST}$ reviewed and revised the manuscript. All authors contributed to the article and approved the submitted version.

\section{FUNDING}

This study was supported by the National Natural Science Foundation of China (81874383, 82074267, 82174185), the Chinese Medicine Scientific Research Project of the Health Commission of Hubei Province (ZY2021Q024), and the program of Tongji-Rongcheng Center for Biomedicine (HUST).

5. Scarsi M, Zanotti C, Chiarini M, Imberti L, PiantoniS, Frassi M, et al. Reduction of Peripheral Blood T Cells Producing IFN- $\gamma$ and IL-17 After Therapy With Abatacept for Rheumatoid Arthritis. Clin Exp Rheumatol (2014) 32:204-10.

6. McInnes IB, Leung BP, Sturrock RD, Field M, Liew FY. Interleukin-15 Mediates T Cell-Dependent Regulation of Tumor Necrosis Factor-Alpha Production in Rheumatoid Arthritis. Nat Med (1997) 3:189-95. doi: 10.1038/ nm0297-189

7. Smolen JS, Aletaha D, Redlich K. The Pathogenesis of Rheumatoid Arthritis: New Insights From Old Clinical Data? Nat Rev Rheumatol (2012) 8:235-43. doi: 10.1038/nrrheum.2012.23

8. Pflanz S, Timans JC, Cheung J, Rosales R, Kanzler H, Gilbert J, et al. IL-27, a Heterodimeric Cytokine Composed of EBI3 and P28 Protein, Induces Proliferation of Naive CD4+ T Cells. Immunity (2002) 16:779-90. doi: 10.1016/s1074-7613(02)00324-2 
9. Paradowska-Gorycka A, Raszkiewicz B, Jurkowska M, Felis-Giemza A, Romanowska-Prochnicka K, Mańczak M, et al. Association of Single Nucleotide Polymorphisms in the IL27 Gene With Rheumatoid Arthritis. Scand J Immunol (2014) 80:298-305. doi: 10.1111/sji.12209

10. Wong CK, Chen DP, Tam LS, Li EK, Yin YB, Lam CW. Effects of Inflammatory Cytokine IL-27 on the Activation of Fibroblast-Like Synoviocytes in Rheumatoid Arthritis. Arthritis Res Ther (2010) 12:R129. doi: 10.1186/ar3067

11. Tanida S, Yoshitomi H, Ishikawa M, Kasahara T, Murata K, Shibuya H, et al. IL-27-Producing CD14(+) Cells Infiltrate Inflamed Joints of Rheumatoid Arthritis and Regulate Inflammation and Chemotactic Migration. Cytokine (2011) 55:237-44. doi: 10.1016/j.cyto.2011.04.020

12. Millier MJ, Lazaro K, Stamp LK, Hessian PA. The Contribution From Interleukin-27 Towards Rheumatoid Inflammation: Insights From Gene Expression. Genes Immun (2020) 21:249-59. doi: 10.1038/s41435-020-0102-z

13. Yoshida H, Hamano S, Senaldi G, Covey T, Faggioni R, Mu S, et al. WSX-1 Is Required for the Initiation of Th1 Responses and Resistance to L. Major Infection. Immunity (2001) 15:569-78. doi: 10.1016/s1074-7613(01)00206-0

14. Vignali DA, Kuchroo VK. IL-12 Family Cytokines: Immunological Playmakers. Nat Immunol (2012) 13:722-8. doi: 10.1038/ni.2366

15. Jones GW, Hill DG, Cardus A, Jones SA. IL-27: A Double Agent in the IL-6 Family. Clin Exp Immunol (2018) 193:37-46. doi: 10.1111/cei.13116

16. Stumhofer JS, Tait ED, Quinn WJ3rd, HoskenN, SpudyB, Goenka R, et al. A Role for IL-27p28 as an Antagonist of Gp130-Mediated Signaling. Nat Immunol (2010) 11:1119-26. doi: 10.1038/ni.1957

17. Chehboun S, Labrecque-Carbonneau J, Pasquin S, Meliani Y, Meddah B, Ferlin W, et al. Epstein-Barr Virus-Induced Gene 3 (EBI3) can Mediate IL-6 TransSignaling. J Biol Chem (2017) 292:6644-56. doi: 10.1074/jbc.M116.762021

18. Min B, Kim D, Feige MJ. IL-30 (IL-27A): A Familiar Stranger in Immunity, Inflammation, and Cancer. Exp Mol Med (2021) 53:823-34. doi: 10.1038/ s12276-021-00630-x

19. Dibra D, Cutrera J, Xia X, Kallakury B, Mishra L, Li S. Interleukin-30: A Novel Antiinflammatory Cytokine Candidate for Prevention and Treatment of Inflammatory Cytokine-Induced Liver Injury. Hepatology (2012) 55:120414. doi: $10.1002 /$ hep. 24814

20. Garbers C, Spudy B, Aparicio-Siegmund S, Waetzig GH, Sommer J, Hölscher C, et al. An Interleukin-6 Receptor-Dependent Molecular Switch Mediates Signal Transduction of the IL-27 Cytokine Subunit P28 (IL-30) via a Gp130 Protein Receptor Homodimer. J Biol Chem (2013) 288:4346-54. doi: 10.1074/ jbc.M112.432955

21. Yoshida H, Hunter CA. The Immunobiology of Interleukin-27. Annu Rev Immunol (2015) 33:417-43. doi: 10.1146/annurev-immunol-032414-112134

22. Jones LL, Vignali DA. Molecular Interactions Within the IL-6/IL-12 Cytokine/Receptor Superfamily. Immunol Res (2011) 51:5-14. doi: 10.1007/ s12026-011-8209-y

23. Perona-Wright G, Kohlmeier JE, Bassity E, Freitas TC, Mohrs K, Cookenham T, et al. Persistent Loss of IL-27 Responsiveness in CD8+ Memory T Cells Abrogates IL-10 Expression in a Recall Response. Proc Natl Acad Sci USA (2012) 109:18535-40. doi: 10.1073/pnas.1119133109

24. Lambert QT, Pradhan A, Roll JD, Reuther GW. Mutations in the Transmembrane and Juxtamembrane Domains Enhance IL27R Transforming Activity. Biochem J (2011) 438:155-64. doi: 10.1042/ bj20110351

25. Pradhan A, Lambert QT, Reuther GW. Transformation of Hematopoietic Cells and Activation of JAK2-V617F by IL-27R, a Component of a Heterodimeric Type I Cytokine Receptor. Proc Natl Acad Sci USA (2007) 104:18502-7. doi: 10.1073/pnas.0702388104

26. Dietrich C, Candon S, Ruemmele FM, Devergne O. A Soluble Form of IL27r $\alpha$ Is a Natural IL-27 Antagonist. J Immunol (2014) 192:5382-9. doi: 10.4049/jimmunol.1303435

27. Swaminathan S, Dai L, Lane HC, Imamichi T. Evaluating the Potential of IL27 as a Novel Therapeutic Agent in HIV-1 Infection. Cytokine Growth Factor $\operatorname{Rev}(2013)$ 24:571-7. doi: 10.1016/j.cytogfr.2013.07.001

28. Pflanz S, Hibbert L, Mattson J, Rosales R, Vaisberg E, Bazan JF, et al. WSX-1 and Glycoprotein 130 Constitute a Signal-Transducing Receptor for IL-27. J Immunol (2004) 172:2225-31. doi: 10.4049/jimmunol.172.4.2225

29. Lin M, Rose-John S, Grötzinger J, Conrad U, Scheller J. Functional Expression of a Biologically Active Fragment of Soluble Gp130 as an ELP-Fusion Protein in Transgenic Plants: Purification via Inverse Transition Cycling. Biochem J (2006) 398:577-83. doi: 10.1042/bj20060544

30. Liu J, Guan X, Ma X. Regulation of IL-27 P28 Gene Expression in Macrophages Through MyD88- and Interferon-Gamma-Mediated Pathways. J Exp Med (2007) 204:141-52. doi: 10.1084/jem.20061440

31. Sonobe Y, Yawata I, Kawanokuchi J, Takeuchi H, Mizuno T, Suzumura A. Production of IL-27 and Other IL-12 Family Cytokines by Microglia and Their Subpopulations. Brain Res (2005) 1040:202-7. doi: 10.1016/ j.brainres.2005.01.100

32. Dibra D, Cutrera JJ, Li S. Coordination Between TLR9 Signaling in Macrophages and CD3 Signaling in T Cells Induces Robust Expression of IL-30. J Immunol (2012) 188:3709-15. doi: 10.4049/jimmunol.1100883

33. Wirtz S, Becker C, Fantini MC, Nieuwenhuis EE, Tubbe I, Galle PR, et al. EBV-Induced Gene 3 Transcription Is Induced by TLR Signaling in Primary Dendritic Cells via NF-Kappa B Activation. J Immunol (2005) 174:2814-24. doi: 10.4049/jimmunol.174.5.2814

34. Ouaked N, Mantel PY, Bassin C, Burgler S, Siegmund K, Akdis CA, et al. Regulation of the Foxp3 Gene by the Th1 Cytokines: The Role of IL-27-Induced STAT1. J Immunol (2009) 182:1041-9. doi: 10.4049/jimmunol.182.2.1041

35. Sprecher CA, Grant FJ, Baumgartner JW, Presnell SR, Schrader SK, Yamagiwa T, et al. Cloning and Characterization of a Novel Class I Cytokine Receptor Biochem Biophys Res Commun (1998) 246:82-90. doi: 10.1006/bbrc.1998.8576

36. Guzzo C, Che Mat NF, Gee K. Interleukin-27 Induces a STAT1/3- and NFkappaB-Dependent Proinflammatory Cytokine Profile in Human Monocytes. J Biol Chem (2010) 285:24404-11. doi: 10.1074/jbc.M110.112599

37. Greenwell-Wild T, Vázquez N, Jin W, Rangel Z, Munson PJ, Wahl SM. Interleukin-27 Inhibition of HIV-1 Involves an Intermediate Induction of Type I Interferon. Blood (2009) 114:1864-74. doi: 10.1182/blood-2009-03211540

38. Lalive PH, Kreutzfeldt M, Devergne O, Metz I, Bruck W, Merkler D, et al. Increased Interleukin-27 Cytokine Expression in the Central Nervous System of Multiple Sclerosis Patients. J Neuroinflamm (2017) 14:144. doi: 10.1186/ s12974-017-0919-1

39. Lai X, Wang H, Cao J, Li Y, Dai Y, Xiang Y, et al. Circulating IL-27 Is Elevated in Rheumatoid Arthritis Patients. Molecules (2016) 21(11):1565. doi: 10.3390/ molecules 21111565

40. Ylitalo R, Heimbürger M, Lindestad PA. Vocal Fold Deposits in Autoimmune Disease-an Unusual Cause of Hoarseness. Clin Otolaryngol Allied Sci (2003) 28:446-50. doi: 10.1046/j.1365-2273.2003.00742.x

41. Kitas G, Banks MJ, Bacon PA. Cardiac Involvement in Rheumatoid Disease. Clin Med (Lond) (2001) 1:18-21. doi: 10.7861/clinmedicine.1-1-18

42. Walker W, Wright V. Pulmonary Lesions and Rheumatoid Arthritis Medicine (1968) 47(6):501-20. doi: 10.1097/00005792-196811000-00003

43. Michet CJ3rd, StrobovaK, Achenbach S, CrowsonCS, Matteson EL. Hospitalization Rates and Utilization Among Patients With Rheumatoid Arthritis: A Population-Based Study From 1987 to 2012 in Olmsted County, Minnesota. Mayo Clin Proc (2015) 90:176-83. doi: 10.1016/ j.mayocp.2014.12.009

44. Nyhäll-Wåhlin BM, Turesson C, Jacobsson LT, Nilsson J, Forslind K, Albertsson K, et al. The Presence of Rheumatoid Nodules at Early Rheumatoid Arthritis Diagnosis Is a Sign of Extra-Articular Disease and Predicts Radiographic Progression of Joint Destruction Over 5 Years. Scand J Rheumatol (2011) 40:81-7. doi: 10.3109/03009742.2010.509103

45. Han M, Zheng Y. Comprehensive Analysis of Single Nucleotide Polymorphisms in Human microRNAs. PloS One (2013) 8:e78028. doi: 10.1371/journal.pone.0078028

46. Yamagata K, Nakayamada S, Tanaka Y. Critical Roles of Super-Enhancers in the Pathogenesis of Autoimmune Diseases. Inflammation Regener (2020) 40:16. doi: 10.1186/s41232-020-00124-9

47. Yan J, Shang X, Rong X. Relationship Between IL-27 Gene Polymorphism and Susceptibility of Rheumatoid Arthritis in Chinese Han Population. Int J Clin Exp Med (2015) 8:6262-6.

48. Niedbala W, Cai B, Wei X, Patakas A, Leung BP, McInnes IB, et al. Interleukin 27 Attenuates Collagen-Induced Arthritis. Ann Rheum Dis (2008) 67:1474-9. doi: 10.1136/ard.2007.083360

49. Pickens SR, Chamberlain ND, Volin MV, Mandelin AM2nd, AgrawalH, Matsui M, et al. Local Expression of Interleukin-27 Ameliorates CollagenInduced Arthritis. Arthritis Rheum (2011) 63:2289-98. doi: 10.1002/art.30324 
50. Cao Y, Doodes PD, Glant TT, Finnegan A. IL-27 Induces a Th1 Immune Response and Susceptibility to Experimental Arthritis. J Immunol (2008) 180:922-30. doi: 10.4049/jimmunol.180.2.922

51. Hashimoto M. Th17 in Animal Models of Rheumatoid Arthritis. J Clin Med (2017) 6(7):73. doi: 10.3390/jcm6070073

52. Finnegan A, Mikecz K, Tao P, Glant TT. Proteoglycan (Aggrecan)-Induced Arthritis in BALB/c Mice Is a Th1-Type Disease Regulated by Th2 Cytokines. J Immunol (1999) 163:5383-90.

53. Hibbert L, Pflanz S, De Waal Malefyt R, Kastelein RA. IL-27 and IFN-Alpha Signal via Stat 1 and Stat 3 and Induce T-Bet and IL-12Rbeta2 in Naive T Cells. J Interferon Cytokine Res (2003) 23:513-22. doi: 10.1089/10799900360708632

54. Owaki T, Asakawa M, Fukai F, Mizuguchi J, Yoshimoto T. IL-27 Induces Th1 Differentiation via P38 MAPK/T-Bet- and Intercellular Adhesion Molecule-1/ LFA-1/ERK1/2-Dependent Pathways. J Immunol (2006) 177:7579-87. doi: 10.4049/jimmunol.177.11.7579

55. Lucas S, Ghilardi N, Li J, de Sauvage FJ. IL-27 Regulates IL-12 Responsiveness of Naive CD4+ T Cells Through Stat1-Dependent and -Independent Mechanisms. Proc Natl Acad Sci U.S.A. (2003) 100:15047-52. doi: 10.1073/pnas.2536517100

56. Liu H, Rohowsky-Kochan C. Interleukin-27-Mediated Suppression of Human Th17 Cells Is Associated With Activation of STAT1 and Suppressor of Cytokine Signaling Protein 1. J Interferon Cytokine Res (2011) 31:459-69. doi: 10.1089/jir.2010.0115

57. Huber M, Steinwald V, Guralnik A, Brüstle A, Kleemann P, Rosenplänter C, et al. IL-27 Inhibits the Development of Regulatory T Cells via STAT3. Int Immunol (2008) 20:223-34. doi: 10.1093/intimm/dxm139

58. Neufert C, Becker C, Wirtz S, Fantini MC, Weigmann B, Galle PR, et al. IL-27 Controls the Development of Inducible Regulatory T Cells and Th17 Cells via Differential Effects on STAT1. Eur J Immunol (2007) 37:1809-16. doi: 10.1002/eji.200636896

59. Cox JH, Kljavin NM, Ramamoorthi N, Diehl L, Batten M, Ghilardi N. IL-27 Promotes T Cell-Dependent Colitis Through Multiple Mechanisms. J Exp Med (2011) 208:115-23. doi: 10.1084/jem.20100410

60. Nguyen QT, Jang E, Le HT, Kim S, Kim D, Dvorina N, et al. IL-27 Targets Foxp3+ Tregs to Mediate Antiinflammatory Functions During Experimental Allergic Airway Inflammation. JCI Insight (2019) 4(2):e123216. doi: 10.1172/ jci.insight. 123216

61. Do J, Kim D, Kim S, Valentin-Torres A, Dvorina N, Jang E, et al. Treg-Specific IL-27r $\alpha$ Deletion Uncovers a Key Role for IL-27 in Treg Function to Control Autoimmunity. Proc Natl Acad Sci USA (2017) 114:10190-5. doi: 10.1073/ pnas. 1703100114

62. Do JS, Visperas A, Sanogo YO, Bechtel JJ, Dvorina N, Kim S, et al. An IL-27/Lag3 Axis Enhances Foxp3+ Regulatory T Cell-Suppressive Function and Therapeutic Efficacy. Mucosal Immunol (2016) 9:137-45. doi: 10.1038/mi.2015.45

63. Batten M, Li J, Yi S, Kljavin NM, Danilenko DM, Lucas S, et al. Interleukin 27 Limits Autoimmune Encephalomyelitis by Suppressing the Development of Interleukin 17-Producing T Cells. Nat Immunol (2006) 7:929-36. doi: $10.1038 /$ ni1375

64. Wright SD. CD14 and Innate Recognition of Bacteria. J Immunol (1995) 155:6-8.

65. Kalliolias GD, Zhao B, Triantafyllopoulou A, Park-Min KH, Ivashkiv LB. Interleukin-27 Inhibits Human Osteoclastogenesis by Abrogating RANKLMediated Induction of Nuclear Factor of Activated T Cells C1 and Suppressing Proximal RANK Signaling. Arthritis Rheum (2010) 62:402-13. doi: 10.1002/art.27200

66. Kamiya S, Okumura M, Chiba Y, Fukawa T, Nakamura C, Nimura N, et al. IL27 Suppresses RANKL Expression in CD4+ T Cells in Part Through STAT3. Immunol Lett (2011) 138:47-53. doi: 10.1016/j.imlet.2011.02.022

67. Hill DG, Ward A, Nicholson LB, Jones GW. Emerging Roles for IL-6 Family Cytokines as Positive and Negative Regulators of Ectopic Lymphoid Structures. Cytokine (2021) 146:155650. doi: 10.1016/j.cyto.2021.155650

68. Jones GW, Bombardieri M, Greenhill CJ, McLeod L, Nerviani A, Rocher-Ros $\mathrm{V}$, et al. Interleukin-27 Inhibits Ectopic Lymphoid-Like Structure Development in Early Inflammatory Arthritis. J Exp Med (2015) 212:1793802. doi: $10.1084 /$ jem.20132307

69. Firestein GS. Evolving Concepts of Rheumatoid Arthritis. Nature (2003) 423:356-61. doi: 10.1038/nature01661
70. Park H, Li Z, Yang XO, Chang SH, Nurieva R, Wang YH, et al. A Distinct Lineage of CD4 T Cells Regulates Tissue Inflammation by Producing Interleukin 17. Nat Immunol (2005) 6:1133-41. doi: 10.1038/ni1261

71. Koenders MI, van den Berg WB. Translational Mini-Review Series on Th17 Cells: Are T Helper 17 Cells Really Pathogenic in Autoimmunity? Clin Exp Immunol (2010) 159:131-6. doi: 10.1111/j.1365-2249.2009.04039.x

72. Wan YY. Regulatory T Cells: Immune Suppression and Beyond. Cell Mol Immunol (2010) 7:204-10. doi: 10.1038/cmi.2010.20

73. Jiang Q, Yang G, Liu Q, Wang S, Cui D. Function and Role of Regulatory T Cells in Rheumatoid Arthritis. Front Immunol (2021) 12:626193. doi: 10.3389/ fimmu.2021.626193

74. Lari R, Kitchener PD, Hamilton JA. The Proliferative Human Monocyte Subpopulation Contains Osteoclast Precursors. Arthritis Res Ther (2009) 11: R23. doi: 10.1186/ar2616

75. Adamopoulos IE, Pflanz S. The Emerging Role of Interleukin 27 in Inflammatory Arthritis and Bone Destruction. Cytokine Growth Factor Rev (2013) 24:115-21. doi: 10.1016/j.cytogfr.2012.10.001

76. Astorri E, Nerviani A, Bombardieri M, Pitzalis C. Towards a Stratified Targeted Approach With Biologic Treatments in Rheumatoid Arthritis: Role of Synovial Pathobiology. Curr Pharm Des (2015) 21:2216-24. doi: 10.2174/1381612821666150310145758

77. Peters A, Pitcher LA, Sullivan JM, Mitsdoerffer M, Acton SE, Franz B, et al. Th17 Cells Induce Ectopic Lymphoid Follicles in Central Nervous System Tissue Inflammation. Immunity (2011) 35:986-96. doi: 10.1016/ j.immuni.2011.10.015

78. Hirota K, Turner JE, Villa M, Duarte JH, Demengeot J, Steinmetz OM, et al. Plasticity of Th17 Cells in Peyer's Patches Is Responsible for the Induction of T Cell-Dependent IgA Responses. Nat Immunol (2013) 14:372-9. doi: $10.1038 /$ ni.2552

79. Miyamoto Y, Uga H, Tanaka S, Kadowaki M, Ikeda M, Saegusa J, et al. Podoplanin Is an Inflammatory Protein Upregulated in Th17 Cells in SKG Arthritic Joints. Mol Immunol (2013) 54:199-207. doi: 10.1016/ j.molimm.2012.11.013

80. Rausch M, Moodley D, Peluso M, Koseoglu S, Hua J, Tan G, et al. Increased Serum Levels of EBI3 Are Associated With Poor Outcome in Hepatocellular Carcinoma Patients and SRF388, a First-in-Class IL-27 Blocking Antibody, Inhibits the Growth of Murine Liver Tumors. J J ImmunoTher Cancer (2020) 8:A435-A. doi: 10.1136/jitc-2020-SITC2020.0727

81. Rausch M, Hua J, Moodley D, White KF, Walsh KH, Miller CE, et al. Increased IL-27 Is Associated With Poor Prognosis in Renal Cell Carcinoma and Supports Use of SRF388, a First-in-Class IL-27p28 Blocking Antibody, to Counteract IL-27-Mediated Immunosuppression in This Setting. J Cancer Res (2020) 80:4550. doi: 10.1158/1538-7445.AM2020-4550

82. Patnaik A, Morgensztern D, Mantia C, Tannir NM, Harshman LC, Hill J, et al. Results of a Phase 1 Study of SRF388, a First-in-Human, First-in-Class, HighAffinity Anti-IL-27 Antibody in Advanced Solid Tumors. J Clin Oncol (2021) 39:2551. doi: 10.1200/JCO.2021.39.15_suppl.2551

Conflict of Interest: The authors declare that the research was conducted in the absence of any commercial or financial relationships that could be construed as a potential conflict of interest.

Publisher's Note: All claims expressed in this article are solely those of the authors and do not necessarily represent those of their affiliated organizations, or those of the publisher, the editors and the reviewers. Any product that may be evaluated in this article, or claim that may be made by its manufacturer, is not guaranteed or endorsed by the publisher.

Copyright (c) 2022 Han, Chen, Yu, Yan, Li, Ba, Lin, Huang, Shen, Huang, Qin, Geng, Liu, Wang and Tu. This is an open-access article distributed under the terms of the Creative Commons Attribution License (CC BY). The use, distribution or reproduction in other forums is permitted, provided the original author(s) and the copyright owner(s) are credited and that the original publication in this journal is cited, in accordance with accepted academic practice. No use, distribution or reproduction is permitted which does not comply with these terms. 